\title{
Caracterização dos pacientes e procedimentos executados no serviço de atendimento a pacientes com necessidades especiais da Faculdade de Odontologia de Araraquara - UNESP
}

\author{
Characterization of patients and procedures performed in the service to patients with \\ special needs of Araraquara School of Dentistry - UNESP
}
Natália Bertolo DOMINGUESa, Kelly Caroline Mello AYRES ${ }^{a}$, Matheus Racy MARIUSSO ${ }^{a}$, Ângela Cristina Cilense ZUANONª, Elisa Maria Aparecida GIRO ${ }^{\text {a* }}$

aFaculdade de Odontologia, UNESP - Universidade Estadual Paulista "Júlio de Mesquita Filho", Araraquara, SP, Brasil

\begin{abstract}
Resumo
Objetivo: Caracterizar os pacientes atendidos e os procedimentos realizados no Serviço de Atendimento a Pacientes com Necessidades Especiais da Faculdade de Odontologia de Araraquara (FOAr) - UNESP. Metodologia: A partir dos prontuários odontológicos dos pacientes atendidos em nível ambulatorial entre 2007 e 2013, foram obtidas informações em relação a idade no início do tratamento, tipo de deficiência, medicamentos usados, número de sessões de atendimento e número e tipo de procedimentos odontológicos realizados. Resultado: Dos 232 prontuários analisados, observou-se que mais da metade dos pacientes $(56,0 \%)$ apresentava deficiência mental, paralisia cerebral ou anomalias congênitas determinadas por agentes infecciosos, com deficiência mental associada. Entre as anomalias congênitas, a mais prevalente foi a Síndrome de Down (9,9\%). Apenas $20 \%$ dos pacientes iniciaram o tratamento antes dos dez anos de idade e 62,0\% faziam uso de anticonvulsivantes, antipsicóticos, ansiolíticos, antiepilépticos e antidepressivos. Foram realizados 4.506 procedimentos odontológicos, sendo $37,6 \%$ preventivos e $62,4 \%$ curativos (Restauradores, 28,0\%; Periodontais, 13,9\%; Endodônticos, 9,7\%; Cirúrgicos, 9,1\%; Protéticos, 1,7\%). Observou-se também que, para a maior parte dos pacientes (63\%), foram necessárias até dez sessões para a realização do tratamento. Conclusão: Os pacientes que procuraram tratamento odontológico no Serviço de Atendimento a Pacientes com Necessidades Especiais da FOAr apresentavam, na sua maioria, deficiências com envolvimento neurológico. Apesar de esse serviço priorizar os procedimentos preventivos, a maior parte dos procedimentos executados foi curativa, $\mathrm{o}$ que está diretamente relacionado com a procura tardia pelo tratamento odontológico.
\end{abstract}

Descritores: Pessoas com deficiência; assistência odontológica para pessoas com deficiências; uso de medicamentos.

\begin{abstract}
Objective: To characterize the patients and dental procedures performed in Service to Patients with Special Needs of Araraquara School of Dentistry (FOAr) - UNESP. Methodology: From the dental records of patients treated at outpatient basis between 2007 and 2013, information regarding age at the beginning of treatment, type of disability, controlled medications used, number of dental sessions, and number and type of dental procedures performed was obtained. Result: Of the 232 records analyzed, more than half of the patients (56.0\%) had mental retardation, cerebral palsy or congenital abnormalities caused by infectious agents associated with mental retardation. Among the congenital abnormalities, the most prevalent was the Down syndrome (9.9\%). Only $20 \%$ of patients started treatment before the age of ten and $62.0 \%$ used anticonvulsants, antipsychotics, anxiolytics, antidepressants and antiepileptics. A total of 4,506 dental procedures were performed, $37.6 \%$ were preventive procedures and $62.4 \%$ curative procedures (restorative, 28.0\%; periodontal, 13.9\%; endodontic, 9.7\%; surgical, 9.1\%, and prosthetic 1.7\%). Also was observed that for most patients (63\%) up to 10 sessions were required for the completion of treatment. Conclusion: Most patients with special needs who sought dental treatment at FOAr presented neurological involvement. Despite this service prioritize preventive procedures; most of the procedures carried out were curative, fact directly linked to late search for the dental treatment.
\end{abstract}

Descriptors: Disabled persons; dental care for disabled; drug utilization. 


\section{INTRODUÇÃO}

O termo "pacientes com necessidades especiais" se refere a pessoas que, por apresentarem algum tipo de desvio da normalidade de ordem física, mental, sensorial, comportamental e/ou de crescimento, precisam receber cuidado diferenciado por um período ou durante a vida toda ${ }^{1}$.

Apesar de a especialidade odontológica voltada ao atendimento de pacientes especiais ter sido regulamentada há algum tempo, ainda são observadas dificuldades de atendimento público para esses indivíduos, visto que, na maioria das vezes, este serviço é oferecido por instituições filantrópicas ou por unidades de atendimento de urgências. De acordo com a Portaria n. ${ }^{0}$ 1060/GM ${ }^{2}$, de 5 de junho de 2002, a atenção integral à saúde das pessoas com deficiência inclui a saúde bucal e a assistência odontológica, devendo o atendimento ser realizado em regime ambulatorial especial ou em regime de internação, quando a natureza da sequela assim o exigir. Entretanto, ainda hoje, pode ser observada inadequação da estrutura física nos locais de atendimento, a qual dificulta o acesso ao tratamento, bem como falta de profissionais com preparo científico, técnico e emocional para o atendimento desses indivíduos.

Alguns cursos de Odontologia incluíram na sua grade curricular uma disciplina voltada para o atendimento odontológico de pacientes com necessidades especiais, objetivando preparar adequadamente os futuros profissionais para o atendimento dessa parcela da população ${ }^{3}$. O atendimento de pacientes com necessidades especiais por estudantes do curso de graduação proporciona o aprendizado não apenas de técnicas para o atendimento odontológico preventivo e curativo, mas procura, principalmente, dar embasamento científico para que estes atuem com segurança quando se depararem com os diferentes problemas neuropsicomotores e/ou sistêmicos apresentados por esses pacientes. Além disso, prepara o futuro profissional para oferecer um tratamento mais humanizado, incentivando uma maior relação interpessoal entre profissional, paciente e responsáveis/cuidadores ${ }^{4}$.

O Departamento de Clínica Infantil da Faculdade de Odontologia de Araraquara (FOAr) - UNESP conta com um grupo de docentes capacitados, que atuam na disciplina optativa "Odontologia para Pacientes com Necessidades Especiais” e no Projeto de Extensão Universitária "Serviço Odontológico para Pacientes Portadores de Necessidades Especiais", preparando estudantes de graduação e de pós-graduação, para oferecer atendimento odontológico de qualidade a indivíduos com necessidade de cuidados especiais de Araraquara e região.

Assim, é de grande importância caracterizar esse grupo e as suas principais necessidades odontológicas, para proporcionar ao estudante um aprendizado diferenciado e de qualidade, que venha ao encontro das necessidades dessa população ${ }^{5}$. Portanto, o presente trabalho objetivou descrever o perfil dos pacientes com necessidade de cuidados especiais atendidos na Faculdade de Odontologia de Araraquara quanto ao tipo de deficiência e aos medicamentos usados, bem como apresentar um levantamento do número e do tipo de procedimentos odontológicos realizados.

\section{METODOLOGIA}

Este estudo observacional do tipo transversal teve a aprovação do Comitê de Ética em Pesquisa em Seres Humanos (Processo 83/2011). Foram avaliados os prontuários odontológicos dos indivíduos atendidos em nível ambulatorial na Disciplina "Odontologia para Pacientes com Necessidades Especiais” e no Projeto de Extensão Universitária "Serviço odontológico para Pacientes Portadores de Necessidades Especiais", do Departamento de Clínica Infantil da Faculdade de Odontologia de Araraquara - UNESP, no período de janeiro de 2007 a dezembro de 2013.

A coleta de dados foi realizada por dois examinadores devidamente treinados. Os seguintes dados foram anotados: idade do paciente no primeiro atendimento, classificação segundo os desvios apresentados, medicamento de uso contínuo utilizado, número e tipo de procedimentos odontológicos realizados, e número de sessões necessárias para completar o tratamento odontológico.

Os quadros de necessidades especiais foram classificados segundo o tipo de comprometimento e/ou as áreas comprometidas pela patologia, seguindo a orientação da International Association for Disabilities and Oral Health (IADH), com adaptação feita por Mugayar ${ }^{1}$ : desvios de inteligência, defeitos físicos, defeitos congênitos, desvios comportamentais, desvios psíquicos, deficiências sensoriais e de audiocomunicação, doenças sistêmicas crônicas, doenças endócrino-metabólicas, desvios sociais e estados fisiológicos especiais.

Os procedimentos odontológicos executados foram agrupados em sete categorias:

1. Procedimentos para diagnóstico: exame clínico da cavidade bucal e exame radiográfico;

2. Procedimentos Preventivos: instrução de higiene bucal e dieta, profilaxia dental, selantes de fossas e fissuras, aplicação tópica de flúor e clorexidina;

3. Procedimentos Periodontais: raspagem supra e subgengival, polimento dental, gengivectomia, gengivoplastia;

4. Procedimentos Restauradores: Restaurações estéticas e funcionais;

5. Procedimentos Endodônticos: proteção pulpar direta, pulpotomia/pulpectomia de dente decíduo ou permanente, curativo intracanal, obturação de câmara coronária e/ou canais radiculares, e retratamento de canais radiculares;

6. Procedimentos Cirúrgicos: biópsia, excisão de lesão de tecidos moles bucais, exodontia de dentes decíduos, permanentes ou supranumerários, exodontia de raiz residual e pós-operatório;

7. Procedimentos Protéticos: Confecção e instalação de prótese fixa, prótese parcial removível e prótese total.

Os dados coletados dos prontuários foram organizados em planilhas e os resultados foram expressos de forma descritiva e apresentados por meio de tabelas. 


\section{RESULTADO}

Os prontuários de 232 pacientes foram avaliados. Cabe ressaltar que, em média, $80 \%$ dos pacientes, depois de finalizado o tratamento inicial proposto, retornaram anualmente as clínicas de atendimento para reavaliações e manutenção do tratamento executado.

Com relação ao perfil dos pacientes atendidos, observou-se que $56,0 \%$ apresentavam deficiência mental, paralisia cerebral ou alterações congênitas determinadas por agentes infecciosos (rubéola, sarampo, toxoplasmose, citomegalovírus e meningite), geralmente com sério envolvimento neuropsicomotor. Os outros 44,0\% apresentaram outros tipos de alterações e/ou síndromes, podendo a deficiência mental estar presente ou não. Entre as anomalias congênitas, a mais prevalente foi a Síndrome de Down $(9,9 \%)$ (Tabela 1). Ainda com relação ao perfil dos pacientes especiais atendidos na FOAr, constatou-se que, com relação à procura pelo primeiro atendimento na instituição, os mesmos encontravam-se na faixa etária de 3 a 60 anos (22,1 $\pm 13,2): 20,0 \%$ na primeira década de vida, seguidos de $23,0 \%$ e $28,0 \%$ na segunda e terceira décadas de vida, respectivamente. Do total, $29,0 \%$ estavam em idades que variaram de 31 a 60 anos.

A maioria dos pacientes (62,0\%) fazia uso contínuo ou frequente de medicamentos, sendo os anticonvulsivantes, antipsicóticos, ansiolíticos, antiepilépticos e antidepressivos aqueles usados com maior frequência; note-se que grande número deles (47,0\%) usava dois ou mais tipos de medicamento (Tabela 2).

No período dos sete anos compreendidos pelo estudo, foi realizado um total de 4.506 procedimentos odontológicos. A maior parte destes foi de procedimentos curativos $(62,4 \%)$ (Tabela 3$)$.

A maioria dos pacientes $(63,0 \%)$ necessitou de até dez sessões de atendimento para a realização do tratamento. Uma parcela considerável destes $(37,0 \%)$ necessitou de um maior número de sessões.

Tabela 1. Classificação dos pacientes atendidos no Projeto de Extensão "Serviço Odontológico para Pacientes Portadores de Necessidades Especiais" e na disciplina "Odontologia para Pacientes com Necessidades Especiais" da Faculdade de Odontologia de Araraquara - UNESP, no período de 2007 a 2013, de acordo com o tipo de deficiência

\begin{tabular}{|c|c|c|c|}
\hline Classificação & Tipo de Deficiência & Número de pacientes (\%) & Total (\%) \\
\hline Desvios da inteligência & - Retardo mental & $50(21,55)$ & $50(21,55)$ \\
\hline \multirow{4}{*}{ Defeitos Físicos } & - Paralisia Cerebral & $48(20,70)$ & \multirow{4}{*}{$56(24,14)$} \\
\hline & - Deficiência física & $4(1,72)$ & \\
\hline & - Osteogênese imperfeita & $1(0,43)$ & \\
\hline & - Encefalocele/mielomeningocele & $3(1,30)$ & \\
\hline \multirow{9}{*}{ Defeitos congênitos } & $\begin{array}{l}\text { - Sequelas de Rubéola/Sarampo/Toxoplasmose/ } \\
\text { Citomegalovírus/Meningite }\end{array}$ & $32(13,80)$ & \multirow{9}{*}{$83(35,77)$} \\
\hline & - Síndrome de Down & $23(9,91)$ & \\
\hline & - Microcefalia & $13(5,60)$ & \\
\hline & - Hidrocefalia & $9(3,90)$ & \\
\hline & - Síndrome de Schwartz Jampel & $2(0,86)$ & \\
\hline & - Síndrome de Turner & $1(0,43)$ & \\
\hline & - Síndrome de Rett & $1(0,43)$ & \\
\hline & - Cromossomo $13 \mathrm{em}$ anel & $1(0,43)$ & \\
\hline & - Macrocefalia & $1(0,43)$ & \\
\hline Desvios comportamentais & - Autismo & $8(3,45)$ & $8(3,45)$ \\
\hline \multirow{2}{*}{$\begin{array}{l}\text { Deficiências sensoriais e de } \\
\text { audiocomunicação }\end{array}$} & - Deficiência visual & $2(0,86)$ & \multirow{2}{*}{$4(1,72)$} \\
\hline & - Deficiência de fonação & $2(0,86)$ & \\
\hline \multirow{3}{*}{ Doenças sistêmicas crônicas } & - Epilepsia ou Transtornos convulsivos & $16(6,90)$ & \multirow{3}{*}{$23(9,92)$} \\
\hline & - Cardiopatias & $5(2,16)$ & \\
\hline & - Pneumopatias & $2(0,86)$ & \\
\hline Doenças endócrino metabólicas & - Distúrbios metabólicos (Diabetes/Hipotireoidismo) & $5(2,16)$ & $5(2,16)$ \\
\hline Sem diagnóstico & & $3(1,29)$ & $3(1,29)$ \\
\hline TOTAL & & $232(100)$ & $232(100)$ \\
\hline
\end{tabular}


Tabela 2. Distribuição dos pacientes atendidos no Projeto de Extensão "Serviço Odontológico para Pacientes Portadores de Necessidades Especiais" e na disciplina "Odontologia para Pacientes com Necessidades Especiais" da Faculdade de Odontologia de Araraquara - UNESP, no período de 2007 a 2013, de acordo com o uso e o tipo de medicamento

\begin{tabular}{lcc}
\hline \multicolumn{1}{c}{ Uso de Medicamento } & Número de Pacientes & Porcentagem (\%) do total de pacientes \\
\hline Não usa & 88 & 37,9 \\
Usa um tipo & 35 & 15,1 \\
\hline Usa dois ou mais tipos & 109 & 47,0 \\
\hline \multicolumn{1}{c}{ Tipo de medicamento } & Número de Pacientes & Porcentagem (\%) dos que usam \\
\hline Anticonvulsivante & 81 & 56,2 \\
\hline Antipsicótico & 52 & 36,1 \\
\hline Ansiolítico & 48 & 33,3 \\
\hline Antiepiléptico & 24 & 16,7 \\
\hline Antidepressivo & 10 & 6,9 \\
Outros & 38 & 26,4 \\
\hline
\end{tabular}

Tabela 3. Número e porcentagem (\%) de procedimentos odontológicos realizados no período de 2007 a 2013, no Projeto de Extensão "Serviço Odontológico para Pacientes Portadores de Necessidades Especiais" e na disciplina "Odontologia para Pacientes com Necessidades Especiais" da Faculdade de Odontologia de Araraquara - UNESP, de acordo com o ano de atendimento e o tipo de procedimento

\begin{tabular}{ccccccccc}
\hline Procedimentos & $\mathbf{2 0 0 7}$ & $\mathbf{2 0 0 8}$ & $\mathbf{2 0 0 9}$ & $\mathbf{2 0 1 0}$ & $\mathbf{2 0 1 1}$ & $\mathbf{2 0 1 2}$ & $\mathbf{2 0 1 3}$ & Total \\
\hline Preventivos & $214(28,8)$ & $225(41,1)$ & $285(43,7)$ & $79(19,8)$ & $326(37,3)$ & $315(43,1)$ & $249(44,1)$ & $1693(37,6)$ \\
Periodontais & $73(9,8)$ & $70(12,8)$ & $81(12,4)$ & $69(17,3)$ & $94(10,7)$ & $145(19,8)$ & $93(16,5)$ & $625(13,9)$ \\
\hline Restauradores & $245(33,0)$ & $192(35,1)$ & $142(21,8)$ & $118(29,6)$ & $268(30,6)$ & $162(22,2)$ & $136(24,1)$ & $1263(28,0)$ \\
Endodônticos & $128(17,3)$ & $30(5,5)$ & $54(8,3)$ & $43(10,8)$ & $100(11,4)$ & $39(5,3)$ & $42(7,4)$ & $436(9,7)$ \\
Cirúrgicos & $74(10,0)$ & $21(3,8)$ & $82(12,6)$ & $56(14,1)$ & $77(8,8)$ & $64(8,7)$ & $38(6,7)$ & $412(9,1)$ \\
Protéticos & $8(1,1)$ & $9(1,7)$ & $5(0,7)$ & $33(8,3)$ & $10(1,1)$ & $6(0,8)$ & $6(1,1)$ & $77(1,7)$ \\
Total & 742 & 547 & 649 & 398 & 875 & 731 & 564 & 4506 \\
\hline
\end{tabular}

\section{DISCUSSÃO}

Fatores como higiene bucal deficiente, condição socioeconômica menos favorecida, respiração bucal, anomalias de oclusão, dieta cariogênica e uso de medicamentos são comuns em pacientes com deficiência física/mental. Essa realidade pode determinar índices expressivos das doenças cárie e periodontal, as quais podem exercer influência negativa sobre a qualidade de vida desses indivíduos ${ }^{6-10}$.

Como pode ser observado na Tabela 1, dos pacientes com necessidades de cuidados especiais atendidos no Projeto de Extensão Universitária "Serviço odontológico para pacientes portadores de necessidades especiais" e na Disciplina "Odontologia para Pacientes com Necessidades Especiais”, a grande maioria apresentava deficiência mental. Esse quadro era esperado, uma vez que ambos os serviços priorizam o atendimento de pacientes com problemas neuropsicomotores. Pereira et al. ${ }^{3}$ verificaram $55,8 \%$ de pacientes com distúrbios neurológicos entre os pacientes atendidos no seu programa e ressaltaram que, muitas vezes, a classificação desses indivíduos em uma determinada categoria é bastante difícil, pois grande parte deles apresenta mais de uma alteração. Além disso, muitos pacientes vêm encaminhados por instituições que atendem tipos específicos de deficiência, o que pode influenciar os resultados desta análise.

Entre os indivíduos atendidos nas clínicas da FOAr, 20,7\% apresentavam paralisia cerebral, condição caracterizada por uma lesão cerebral não progressiva, com alterações neuromusculares e esqueléticas ${ }^{11}$. A elevada prevalência da cárie dental em pacientes com paralisia cerebral ${ }^{12} \mathrm{e}$, consequentemente, a alta necessidade de tratamento odontológico curativo, pode estar ligada a diferentes fatores, como a severidade da paralisia cerebral e o nível do retardo mental. ${ }^{6}$ Tais condições os tornam mais dependentes no momento da alimentação, determinam modificação no padrão de dieta alimentar e limitam o controle do biofilme ${ }^{13}$. Além disso, os pacientes com paralisia cerebral apresentam baixo fluxo e baixa capacidade tampão salivares ${ }^{6,14,15}$, fatores que estão relacionados com o aumento do risco à cárie.

Aproximadamente $10 \%$ dos pacientes atendidos apresentavam Síndrome de Down, a qual está relacionada com limitações no desenvolvimento físico e mental, e com deficiência imunológica ${ }^{16,17}$. Esta última, associada à má higiene bucal que esses indivíduos 
apresentam, determina o aparecimento precoce da doença periodontal, que se agrava com a idade ${ }^{18}$.

Indivíduos com alterações neurológicas geralmente apresentam redução do fluxo e do $\mathrm{pH}$ salivares, em virtude da utilização sistemática de medicamentos, como os anticonvulsivantes, antipsicóticos, ansiolíticos, antiepilépticos e antidepressivos ${ }^{19}$. No presente trabalho, constatou-se que $62,1 \%$ dos pacientes faziam uso contínuo de um ou mais desses medicamentos. A hipossalivação causada por esses medicamentos leva a alterações na composição eletrolítica e orgânica da saliva, reduzindo a sua capacidade tampão, com consequente aumento da perda mineral da estrutura dentária e comprometimento da remineralização, podendo aumentar o risco à cárie ${ }^{20}$. Além disso, mecanismos de defesa exercidos pela saliva podem ser comprometidos como consequência da hipossalivação ${ }^{21}$. Alguns desses medicamentos podem, também, atuar promovendo um aumento gengival que, associado à má higiene bucal, propicia a inflamação e o desenvolvimento da doença periodontal precoce ${ }^{22}$.

Embora as Clínicas da FOAr priorizem a prevenção da cárie e da doença periodontal, o estudo mostrou que apenas 37,6\% dos procedimentos executados nos pacientes especiais foram preventivos. A maioria $(62,4 \%)$ foi de procedimentos curativos (restauradores, periodontais, endodônticos, cirúrgicos e protéticos). Isso ocorre devido ao fato de os pacientes com necessidades especiais normalmente apresentarem índices de saúde bucal mais precários que a população de maneira geral ${ }^{23}$, devido a comprometimentos inerentes à sua deficiência. Além disso, muitas vezes a demora na procura de tratamento impede uma atuação mais precoce do profissional e, consequentemente, resulta em necessidades de tratamento curativo acumuladas. Como $21,5 \%$ dos pacientes especiais que procuraram atendimento apresentavam retardo mental, 20,7\% apresentavam paralisia cerebral e 35,7\% apresentavam alterações congênitas com sério envolvimento neurológico e motor, quase sempre, eles precisam de cuidadores que passam a ser responsáveis pelos cuidados com a higiene bucal ${ }^{24}$. Contudo, é importante ressaltar que devido à falta de controle e coordenação dos movimentos da mandíbula, dos lábios e da língua que eles apresentam, existe muita dificuldade e até certa relutância por parte dos familiares e/ou responsáveis em promover uma higiene bucal adequada. Esse quadro é agravado pelos seguintes aspectos: carência de programas de treinamento para cuidadores; profissionais pouco qualificados e despreparados para este tipo de atendimento; custo financeiro; desconhecimento dos pais quanto às possibilidades de tratamento, e priorização do tratamento de outros problemas sistêmicos.

Neste trabalho, apenas 20,0\% dos pacientes que procuraram atendimento estavam na primeira década de vida, quando os problemas bucais são de mais fácil solução, os cuidados preventivos podem ser instituídos com maior eficácia e a orientação e a motivação dos cuidadores são mais efetivas. Em idades mais avançadas, como foi observado neste estudo, as necessidades odontológicas exigem tratamentos curativos mais extensos e, muitas vezes, mutiladores. Os dados mostraram, ainda, que apesar da preocupação em se adotarem e incentivarem as medidas preventivas da cárie e da doença periodontal entre estes indivíduos e seus cuidadores, é muito difícil atingir um índice de sucesso adequado. Isso ocorre devido principalmente à falta de colaboração e às dificuldades inerentes a cada tipo de deficiência. Mesmo aqueles pacientes de retorno, atendidos em dois ou mais anos da pesquisa, ainda apresentam a necessidade de uma odontologia curativa.

Vale salientar que, com o aumento da expectativa de vida dos pacientes especiais, dentistas e higienistas se tornam cada vez mais responsáveis pela manutenção da saúde bucal dessa população, por intermédio da prestação de cuidados continuados, desde a infância e por toda a vida, contribuindo, assim, para uma melhora na qualidade de vida dessas pessoas ${ }^{25}$. Isso exige a formação de profissionais mais bem preparados para superar dificuldades específicas geradas pela condição do paciente; além disso, é essencial que haja o comprometimento do núcleo familiar ou dos cuidadores com o comparecimento nas sessões de atendimento e, principalmente, com os cuidados diários para a manutenção da sua saúde bucal.

\section{CONCLUSÃO}

De acordo com os prontuários odontológicos analisados, os pacientes que procuraram tratamento odontológico no Serviço de Atendimento a Pacientes com Necessidades Especiais da FOAr apresentavam, na sua maioria, deficiências com envolvimento neurológico. Apesar de esse serviço priorizar os procedimentos preventivos, a maior parte dos procedimentos executados foi curativa, o que está diretamente relacionado com a procura tardia pelo tratamento odontológico.

\section{AGRADECIMENTOS}

Os autores agradecem aos Estagiários do Departamento de Clínica Infantil, aos Pós-Graduandos do Programa de Pós-Graduação em Ciências Odontológicas e aos Docentes da Disciplina de Odontopediatria - Cyneu Aguiar Pansani, Fábio Cesar Braga de Abreu e Lima, Fernanda Lourenção Brighenti, Fabiano Jeremias, Josimeri Hebling, Lourdes Aparecida Martins dos Santos Pinto e Rita de Cássia Loiola Cordeiro - que participaram do atendimento e da supervisão dos trabalhos executados no Projeto de Extensão Universitária "Serviço odontológico para Pacientes Portadores de Necessidades Especiais" e na Disciplina "Odontologia para Pacientes com Necessidades Especiais", no período de 2007 a 2013.

\section{REFERÊNCIAS}

1. Mugayar LRF. Pacientes portadores de necessidades especiais: manual de odontologia e saúde oral. São Paulo: Pancast; 2000.

2. Brasil. Ministério da Saúde. Portaria n. ${ }^{\circ}$ 1060/GM/MS, 5 de junho de 2002. Diário Oficial da União. Brasília, 5 junho 2002. [citado 2015 Fev 2]. Disponível em: http://www.saude.mg.gov.br/images/documentos/portaria_1060.pdf

3. Pereira LM, Mardero E, Ferreira SH, Kramer PF, Cogo RB. Atenção odontológica em pacientes com deficiências: a experiência do curso de Odontologia da ULBRA (Canoas/RS). Stomatos. 2010;16(31):92-9. 
4. Gomes MJ, Caxias FP, Margon CD, Rosa RG, Carvalho RB. A percepção dos docentes do Curso de Odontologia da UFES em relação à necessidade de inclusão da disciplina denominada "Atendimento Odontológico a Pacientes Portadores de Necessidades Especiais". Rev Bras Pesqui Saúde. 2009;11(1):33-9.

5. Silva ZCM, Pagnoncelli SD, Weber JBB, Fritscher AMG. Avaliação do perfil dos pacientes com necessidades especiais da clínica de odontopediatria da faculdade de odontologia da PUCRS. Rev Odonto Ciênc. 2005;20(50):313-8.

6. Santos MT, Masiero D, Simionato MR. Risk factors for dental caries in children with cerebral palsy. Spec Care Dentist. 2002 May-Jun;22(3):103-7. http://dx.doi.org/10.1111/j.1754-4505.2002.tb01171.x. PMid:12240889.

7. Santos MT, Nogueira MLG. Infantile reflexes and their effects on dental caries and oral hygiene in cerebral palsy individuals. J Oral Rehabil. 2005 Dez;32(12):880-5. http://dx.doi.org/10.1111/j.1365-2842.2005.01518.x. PMid:16297034.

8. Bhowate R, Dubey A. Dentofacial changes and oral health status in mentally challenged children. J Indian Soc Pedod Prev Dent. 2005 Jun;23(2):71-3. http://dx.doi.org/10.4103/0970-4388.16445. PMid:16012208.

9. Gallarreta FWM, Turssi CP, Palma-Dibb RG, Serra MC. Histórico de saúde: atenção a condições sistêmicas e suas implicações, sobretudo nos fatores de risco de cárie. Rev Odonto Ciênc. 2008 Abr-Jun;23(2):192-6.

10. Vellappally S, Gardens SJ, Al Kheraif AA, Krishna M, Babu S, Hashem M, et al. The prevalence of malocclusion and its association with dental caries among 12-18-year-old disabled adolescents. BMC Oral Health. 2014 Out;14(1):123. http://dx.doi.org/10.1186/1472-6831-14123. PMid:25273325.

11. Santos MTR, Masiero D, Novo NF, Simionato MR. Oral conditions in children with cerebral palsy. J Dent Child (Chic). 2003 Jan-Abr;70(1):40-6. PMid:12762607.

12. Sinha N, Singh B, Chhabra KG, Patil S. Comparison of oral health status between children with cerebral palsy and normal children in India: A case-control study. J Indian Soc Periodontol. 2015 Jan-Fev;19(1):78-82. http://dx.doi.org/10.4103/0972-124X.145800. PMid:25810598.

13. Abanto J, Bortolotti R, Carvalho TS, Alves FBT, Raggio DP, Ciamponi AL. Avaliação dos hábitos alimentares de interesse odontológico em crianças com paralisia cerebral. Rev Inst Ciênc Saúde. 2009 Jul-Set;27(3):244-8.

14. Santos MT, Guaré R, Leite M, Ferreira MC, Nicolau J. Does the neuromotor abnormality type affect the salivary parameters in individuals with cerebral palsy? J Oral Pathol Med. 2010 Nov;39(10):770-4. http://dx.doi.org/10.1111/j.1600-0714.2010.00919.x. PMid:20738750.

15. Santos MT, Guaré RO, Leite MF, Ferreira MC, Durão MS, Jardim JR. Salivary osmolality in individuals with cerebral palsy. Arch Oral Biol. 2010 Nov;55(11):855-60. http://dx.doi.org/10.1016/j.archoralbio.2010.07.016. PMid:20732673.

16. Barroeta O, Nungaray L, Lopez-Osuna M, Armendares S, Salamanca F, Kretschmer RR. Defective monocyte chemotaxis in children with Down's syndrome. Pediatr Res. 1983 Abr;17(4):292-5. http://dx.doi.org/10.1203/00006450-198304000-00013. PMid:6222283.

17. Carsetti R, Valentini D, Marcellini V, Scarsella M, Marasco E, Giustini F, et al. Reduced numbers of switched memory B cells with high terminal differentiation potential in Down syndrome. Eur J Immunol. 2015 Mar;45(3):903-14. http://dx.doi.org/10.1002/eji.201445049. PMid:25472482.

18. Reuland-Bosma W. Proefschriften 25 jaar na dato 35. Parodontale afbraak bij syndroom van Down: een immunologisch probleem. Ned Tijdschr Tandheelkd. 2013 Out;120(10):541-5. http://dx.doi.org/10.5177/ntvt.2013.10.13152. PMid:25026741.

19. Siqueira WL, Santos MT, Elangovan S, Simoes A, Nicolau J. The influence of valproic acid on salivary pH in children with cerebral palsy. Spec Care Dentist. 2007 Mar-Apr.27(2):64-6. http://dx.doi.org/10.1111/j.1754-4505.2007.tb00330.x. PMid:17539222.

20. Bardow A, Nyvad B, Nauntofte B. Relationships between medication intake, complaints of dry mouth, salivary flow rate and composition, and the rate of tooth demineralization in situ. Arch Oral Biol. 2001 May;46(5):413-23. http://dx.doi.org/10.1016/S0003-9969(01)00003-6. PMid:11286806.

21. Dawes C, Pedersen AM, Villa A, Ekstrom J, Proctor GB, Vissink A, et al. The functions of human saliva: a review sponsored by the World Workshop on Oral Medicine VI. Arch Oral Biol. 2015 Jun;60(6):863-74. http://dx.doi.org/10.1016/j.archoralbio.2015.03.004. PMid:25841068.

22. Gusmão ES, Cimões R, Coelho RS, Milhomens JA Fo, Santos RL, Sales GCF. Diagnóstico e tratamento do aumento gengival induzido por drogas. Rev Cir Traumatol Buco-Maxilo-Fac. 2009 Jan-Mar;9(1):59-66.

23. Solanki J, Gupta S, Arya A. Dental caries and periodontal status of mentally handicapped institutilized children. J Clin Diagn Res. 2014 Jul;8(7):ZC25-7. http://dx.doi.org/10.7860/JCDR/2014/8983.4557. PMid:25177632.

24. Costa FAS, Quadrado AVM, Brandão AP, Leme BAP, Carneiro BV, Castanho DLM, et al. Síndrome da Rubéola Congênita: revisão de literatura. Rev Med Saude Brasilia. 2013;2(1):46-57.

25. Dougherty NJ. A review of cerebral palsy for the oral health professional. Dent Clin North Am. 2009 Abr;53(2):329-38. http://dx.doi. org/10.1016/j.cden.2008.12.001. PMid:19269401.

\section{CONFLITOS DE INTERESSE}

Os autores declaram não haver conflitos de interesse.

\section{*AUTOR PARA CORRESPONDÊNCIA}

Prof $^{a}$. Dra . Elisa Maria Aparecida Giro, Departamento de Clínica Infantil, Faculdade de Odontologia de Araraquara, UNESP - Universidade Estadual Paulista “Júlio de Mesquita Filho”, Rua Humaitá, 1680, 1º Andar, Centro, 14801-903 Araraquara - SP, Brasil, e-mail: egiro@foar.unesp.br 